\title{
Solar cycle forecasting: A nonlinear dynamics approach
}

\author{
S. Sello* \\ Mathematical and Physical Models, Enel Research, Via Andrea Pisano 120, 56122 Pisa, Italy
}

Received 1 June 2001 / Accepted 12 July 2001

\begin{abstract}
The problem of prediction of a given time series is examined on the basis of recent nonlinear dynamics theories. Particular attention is devoted to forecast the amplitude and phase of one of the most common solar indicator activities, the international monthly smoothed sunspot number. It is well known that the solar cycle is very difficult to predict due to the intrinsic complexity of the related time behaviour and to the lack of a successful quantitative theoretical model of the Sun's magnetic cycle. Starting from a recent previous work, we checked the reliability and accuracy of a forecasting model based on concepts of nonlinear dynamical systems applied to experimental time series, such as embedding phase space, Lyapunov spectrum, chaotic behaviour. The model is based on a local hypothesis of the behaviour on embedding space, utilising an optimal number of neighbour vectors to predict the future evolution. The performances of this method for the current 23rd solar cycle suggest its valuable insertion in the set of the so-called non-precursor statistical- numerical prediction techniques. The main task is to set up and to compare a promising numerical nonlinear prediction technique, essentially based on an inverse problem, with the most accurate prediction methods, like the so-called "precursor methods" which appear now reasonably accurate in predicting "long-term" Sun activity, with particular reference to "solar" and "geomagnetic" precursor methods based on a solar dynamo theory.
\end{abstract}

Key words. Sun: activity - Sun: magnetic fields

\section{Introduction}

Solar activity forecasting is an important topic for various scientific and technological areas, like space activities related to operations of low-Earth orbiting satellites, electric power transmission lines, high frequency radio communications and geophysical applications. The particles and electromagnetic radiations flowing from solar activity outbursts are also important for long term climate variations and thus it is very important to know in advance the phase and amplitude of the next solar and geomagnetic cycles. Nevertheless, the solar cycle is very difficult to predict on the basis of time series of various proposed indicators, due to high frequency content, noise contamination, high dispersion level and high variability both in phase and amplitude, with intermittent behaviour at different scales. This topic is also complicated by the lack of a quantitative theoretical model of the Sun's magnetic cycle. Many attempts to predict the future behaviour of the solar activity are well documented in the literature. Numerous techniques for forecasting are developed to accurately predict phase and amplitude of future solar cycles, but with limited success. Depending on the nature of the prediction methods

* e-mail: sello@pte.enel.it we can distinguish five class: 1) Curve fitting; 2) Precursor; 3) Spectral; 4) Neural Networks; 5) Climatology.

Apart from precursor methods, the main limitation is the short time interval of reliable extrapolations, as the case of the McNish-Lincoln curve fitting method (1949). In the climatological method we predict the behaviour of the future cycle by a weighted average from the past $N$ cycles, based on the assumption of a some degree of correlation of the phenomenon. A preliminary multivariate stochastic approach, inside this class of methods, was recently investigated (Sello 1999).

A modern class of solar activity prediction methods appears to be reasonably successful in predicting "long range" behaviour, the so-called precursor methods. Precursors are early signs of the magnitude of future solar activity that manifest before clear evidence of the next solar cycle. There are two main kinds of precursor methods: geomagnetic (Thompson 1993) and solar (Schatten et al. 1978, 1993). The basic idea is that if these methods work, they must be based on solar physics, in particular dynamo theory. The precursor methods are correlated with a solar dynamo mechanism, where we suppose that the polar field in the descending phase and minimum of a cycle gives an indication of future fully developed toroidal fields inside the Sun that will drive the next solar activity. 


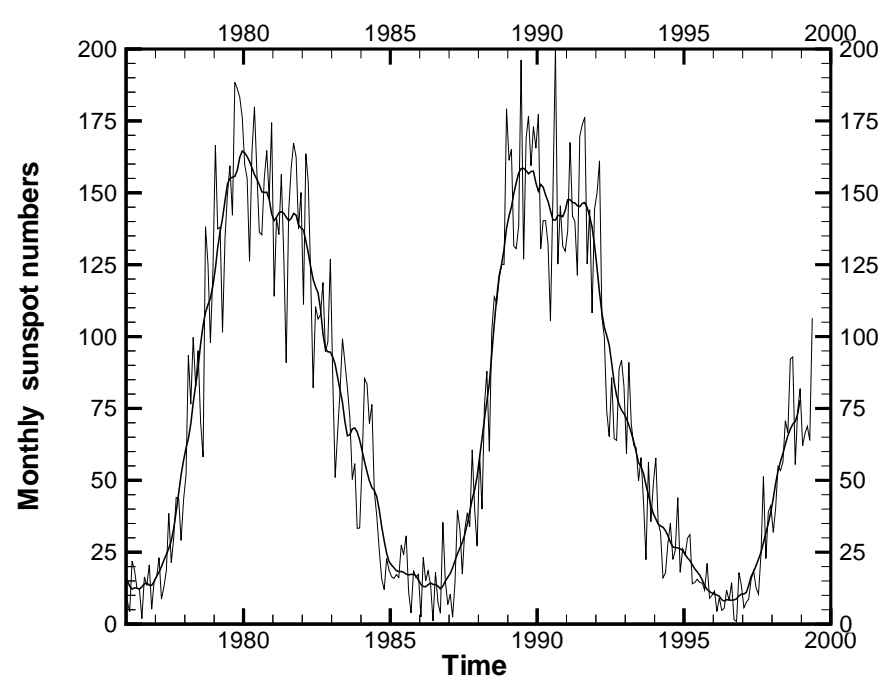

Fig. 1. Monthly mean sunspot numbers, showing high fluctuations and less noisy monthly smoothed sunspot numbers. (from SIDC archive).

The dynamo method was successfully tested using different solar cycles with a proper statistical approach and verified by a scientific panel supported by NOAA Space Environment Center and NASA Office of Space Science (Joselyn et al. 1997). The panel's recommendations for future solar activity studies where based on some criticisms about long term solar cycle predictions including their weak physical foundation and the limitations of the data used to define and extrapolate solar and geophysical behaviour: "prediction research should be supported and the scientific community encouraged to develop a fundamental understanding of the solar cycle that would provide the basis for physical rather than the present empirical prediction methods".

Although the dynamo method and, in general, the precursor methods seems to work well, they might be affected by some severe drawbacks, like secular drifts of nonmagnetic solar wind parameters. However, as pointed out by the authors, we need a better scientific basis. Thus, at present, the statistical numerical approach, based on reliable characterisation and prediction of complex time series behaviour, without any intermediate model, still appears as a valuable technique to provide at least the basis for new insight for future physical prediction methods.

The international sunspot number, $R_{\mathrm{i}}$, is an index characterising the level of solar activity and it is regularly provided by the Solar Influences Data analysis Center (Brussels) of the Federation of Astronomical and Geophysical data analysis Services (SIDC) (Cugnon 1999). The predictions are confined to the so-called monthly smoothed sunspot number, a suitable filtered signal from the monthly sunspot number. Figure 1 shows the time series of the monthly sunspot numbers and the related monthly smoothed sunspot numbers from SIDC data ( $S=\left\langle R_{\mathrm{i}}>\right.$ ) for the last two solar cycles and part of the current 23rd cycle. In order to obtain accurate predictions it is required to analyse the data recorded for the long time. Here we consider the whole time series from a SIDC archive for the monthly mean sunspot numbers covering the period: 1749.5-1999.042. The intrinsic complexity in the behaviour of the sunspot numbers (irregular and intermittent) suggested the possibility of nonlinear (chaotic) dynamics governing the related process, as is well pointed out in many previous works. For example, Spiegel \& Zahn (1996) proposed a chaotic dynamics mechanism at the solar tachocline shear layer to explain the spatio-temporal development of the solar cycle. In particular, we refer here to a recent paper (Zhang 1996) in which we proposed an interesting and promising nonlinear prediction method for the monthly smoothed sunspot numbers. The main aim of the present paper is to support this nonlinear approach adding a more complete and refined analysis through different powerful nonlinear dynamics tools. To this end, we note that detailed wavelet analyses only support the useful assumption of a weak locally stationary condition, at least for scales related to the main long-term component of a highly structured magnetic solar cycle.

\section{Nonlinear dynamics approach}

The nonlinear feature of the monthly mean sunspot number time series was not evident in the past, as is well documented by many different works. For example, in the paper of Price et al. (1992) we found no evidence for the presence of low dimensional deterministic behaviour inside the set of the monthly mean sunspot numbers, suggesting that filtering techniques applied to experimental data in order to obtain smoothed values can give spurious evidence for the presence of deterministic nonlinear behaviour. An earlier work, indicating that the sunspot cycle is indeed chaotic, clearly suggests theoretical limits on the predictability extent of forecasting methods (Mundt et al. 1991). Further accurate investigations on daily time series appeared to confirm that result (Carbonell et al. 1994). Conversely, more recent analyses showed strong evidence for low-dimensional deterministic nonlinear-chaotic dynamics governing sunspot numbers at timescales $T>8$ years (Zhang 1994, 1995; Lawrence et al. 1995; Schreiber 1998). Furthermore, a genuine chaotic behaviour for monthly averaged Wolf numbers, using a nonlinear forecasting method, has been shown by Rozelot (1995). More recently, Kugiumtzis (1999) investigated some properties of a standard and a refined surrogate technique by Prichard \& Theiler (1994) to test the nonlinearity in a real time series, showing that, for the annual sunspot numbers, there is strong evidence that nonlinear dynamics are in fact present, enforcing also the idea that the sunspot numbers are to a first approximation proportional to the squared magnetic field strength. More recently, Li \& Sofia (2001), in order to explain the observed total solar irradiance long-term modulation, proposed a semi-empirical model for the maximum magnetic field $B_{\mathrm{m}}$ in the solar interior which nonlinearly relates the yearly mean sunspot numbers $R_{z}: B_{\mathrm{m}}=B_{0}\left\{A+\left[1+\log _{10}\left(1+R_{z}\right)\right]^{5}\right\}$. 
In the present work we used the method of surrogate data combined with the computation of linear and nonlinear redundancies, in order to show that the monthly mean sunspot numbers contain true nonlinear dependencies (Palus 1995). The use of the information-theoretic functionals, called redundances, has at least three important advantages in comparison to other linear or nonlinear correlation analyses:

1. Various types of redundancies can be constructed in order to test specific types of dependence between or among variables;

2. The redundancies can be naturally evaluated as functions of time lags in order to evaluate dependence structures with respect to dynamics of the system under consideration;

3. For any type of redundancy its linear form exists, which is sensitive to linear dependence only. Linear redundancy is used to test the quality of surrogate data, in order to avoid spurious detection of nonlinearity.

In short, the basic idea in the surrogate data correlation analysis is to compute a linear and nonlinear statistic from data under study (original) and an ensemble of realizations of a linear stochastic process (surrogates) which mimic linear properties only of the original series. If the computed statistic for the original data is significantly different from the values obtained for the surrogate set, one can safely infer that the data were not generated by a linear process; otherwise the null hypothesis that a linear model fully explain the data is accepted and the data can be usefully analysed and characterised by using standard well developed linear methods.

In this work we consider the following nonlinear $R(X, Y)$ redundancy:

$R(X, Y)=H(X)+H(Y)-H(X, Y)$

where:

$$
\begin{aligned}
& H(X)=-\sum_{x \in X} p(x) \log p(x) \\
& H(X, Y)=-\sum_{x \in X} \sum_{y \in Y} p(x, y) \log p(x, y) .
\end{aligned}
$$

In (1) $X$ and $Y$ are random variables with a probability function $p(x) . H(X)$ is the entropy and $H(X, Y)$ is the joint entropy. Here we assume: $Y=X(t+\tau)$ and: $R=$ $R(\tau)$.

If the variables $X$ and $Y$ have zero means, unit variances and correlation matrix $C$, the linear redundancy, $L(X, Y)$ is of the form:

$L(X, Y)=-\frac{1}{2} \sum_{i=1}^{2} \log \left(\sigma_{i}\right)$

where: $\sigma_{i}$ are the eigenvalues of the $2 \times 2$ correlation matrix.

We define the test statistic as the difference between the redundancy obtained for the original data and the

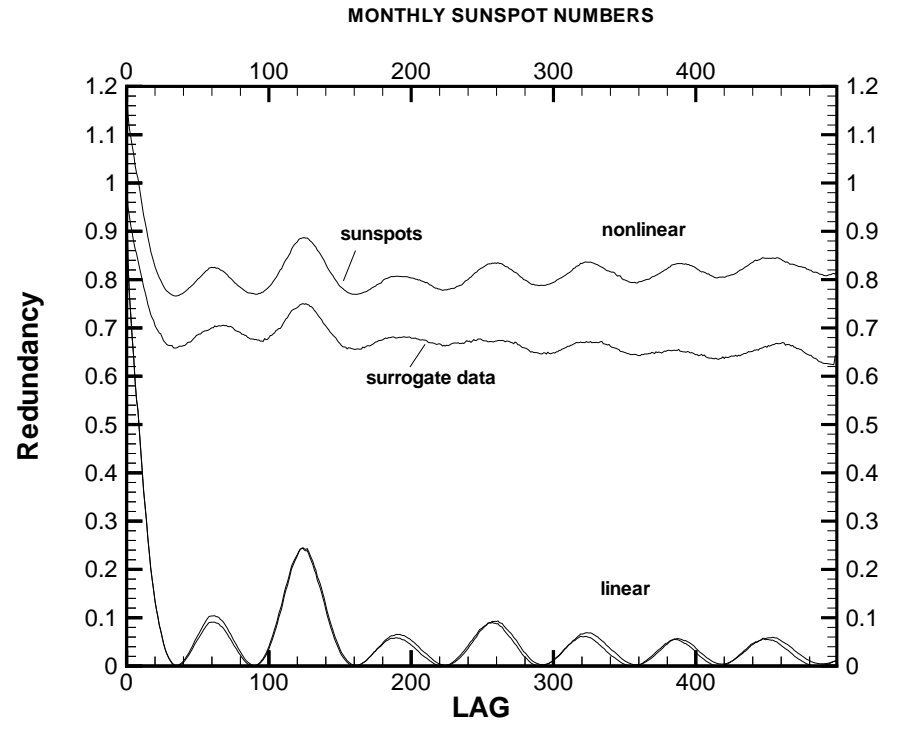

Fig. 2. Linear and nonlinear redundancy analysis: qualitative results. Lag is expressed in months.



Fig. 3. Linear and nonlinear redundancy analysis: quantitative results. The presence of high standard deviations (SD) for the nonlinear case shows the intrinsic nonlinear nature of sunspot numbers. Lag is expressed in months.

mean redundancy of a set of surrogates, in the number of standard deviations (SD) of the latter. Both the redundancies and redundancy-based statistic are functions of time lags $\tau$. General redundancies (1) detect all the dependencies contained in the original data, while the linear redundancies (2) are sensitive to linear structures only. Figure 2 shows the results of the computation of linear and nonlinear redundancies for both the original time series of the monthly mean sunspot numbers and the related surrogate ensemble, as functions of time lags. Here we computed linear and nonlinear redundancies for 30 realisations of 
surrogate time series which mimic the linear properties of the original data. We show the mean redundancies computed for the surrogate ensemble: the linear redundancy curve overlaps with the linear redundancy of the original time series; whereas the general redundancy is distinct from general redundancy of the original data. In Fig. 3 we show the quantitative analysis for the differences between types of redundancies. As expected, linear redundancies for the data and for the surrogates coincide because there is no significant difference in the linear statistic (differences $<1$ SD) i.e. surrogates mimic well the linear dependencies of the data, and should not be a source of spurious results in the nonlinear test. On the other hand, the result for the general redundancy for the original data is clearly different from mean redundancy for surrogates, and the nonlinear statistic indicates highly significant differences. Thus, in this case, the linear stochastic null hypothesis is rejected and, considering also the results from linear statistic, significant nonlinearity is reliably detected inside the original time series.

The nonlinearity analysis on the monthly mean sunspot numbers clearly supports the use of a nonlinear dynamic approach as possible prediction method. Previous preliminary works on the subject show many characteristics of the intrinsic nonlinear dynamics driving sunspot numbers. For example, Ostryakov and Usoskin in 1990 estimated fractal dimension for different periods, finding a value around 4. More recently, Zhang (1995) estimated a fractal dimension: $D=2.8 \pm 0.1$, and the largest Lyapunov exponent: $\lambda=0.023 \mathrm{bits} / \mathrm{month}$ for the monthly mean sunspot numbers in the period: 1850-1992, using the original method by Grassberger \& Procaccia (1983) and Wolf (1985). As consequence there is an upper limit as the time scale for reliable deterministic predictions: $3.6 \pm 0.6$ years. The important indication is that long-term deterministic behaviour of solar cycles is unpredictable. Many authors proposed nonlinear prediction techniques of chaotic time series as an inverse problem for short-term predictions, with different levels of accuracy. Recently, Zhang (1996) proposed a prediction technique which improves medium-term predictions for the monthly smoothed sunspot numbers using a proper local linear map to solve the related inverse problem. The common basis of the above work is the construction of the embedding space from observed data which is the natural vector space in the nonlinear dynamics method. We note also that modern approaches based on neural network predictions are based on embedding space reconstruction in order to set some of the characteristic parameters of the model (Calvo et al. 1995; Kulkarni et al. 1998; Verdes et al. 2000; Fessant et al. 1996).

For typical experimental situations we know only a single scalar time series while the related physical systems possess many essential degrees of freedom. Nonlinear dynamics theory allows the reconstruction of the whole system trajectory in the embedding space, using the method of delay-time.
When a continuous scalar signal $x(t)$, here the monthly mean sunspot numbers, is measured at constant discrete time intervals, $t_{\mathrm{s}}$, we obtain a single scalar time series:

$$
\left\{x\left(t_{0}\right), x\left(t_{0}+t_{\mathrm{s}}\right), x\left(t_{0}+2 t_{\mathrm{s}}\right), \ldots, x\left(t_{0}+N t_{\mathrm{s}}\right)\right\} .
$$

Starting from this time series it is possible to reconstruct a pseudo-trajectory in an $m$-dimensional embedding space through the vectors (embedding vectors):

$$
\begin{aligned}
\boldsymbol{y}_{\mathrm{s}}=\left(x\left(t_{0}+(s-1) t_{\mathrm{s}}\right),\right. & x\left(t_{0}+(s-1) t_{\mathrm{s}}+\tau\right), \ldots \\
& \left.x\left(t_{0}+(s-1) t_{\mathrm{s}}+(m-1) \tau\right)\right)^{T}
\end{aligned}
$$

where: $s=1,2, \ldots,(1-m) \frac{\tau}{t_{\mathrm{s}}}+(1+N)$, and $\tau$ is the delay time.

A selection of proper values for these parameters in the embedding procedure is crucial for the reliability of results, as was pointed out in many works (Shuster 1984; Theiler 1990; Fraser \& Swinney 1986; Abarbanel et al. 1990). Here we use an efficient method to estimate the minimum embedding dimension for a correct reconstruction of the attractor, as introduced by Kennel \& Abarbanel (1994) based on the false neighbors. The main idea is to eliminate "illegal projection", finding, for each embedding vector, the nearest neighbor in different embedding dimensions. When the fraction of false nearest neighbor is less than some threshold, we are able to find the minimum embedding dimension. Figure 4 shows the results of the false neighbor method for the monthly mean sunspot numbers. As is evident, the minimum embedding dimension value is $m=5$. This result is consistent with previous analyses (Zhang 1996), indicating that the sunspot series is related to a low-dimension nonlinear deterministic system, described by a finite number of parameters, or by vectors in a 5-dimensional phase space. Zhang, using the Grassberger-Procaccia method, found saturation for correlation dimension: $d=2.8$ at $m=7$; on the other hand, his prediction technique was based on the value: $m=3$. In Rozelot (1995), using a correlation coefficient and a nonlinear prediction method, we estimated an optimal embedding dimension equal to 3 .

The choice of delay time $\tau$ is here based on the socalled mutual information of Fraser \& Swinney (1986), which is more adequate than an autocorrelation function, when nonlinear dependences are present. Mutual information coincides with the general redundancy given by (1). The computation, for the monthly mean sunspot numbers, indicates a mutual information first local minimum around $40 t_{\mathrm{s}}$, corresponding to a period of 3.32 years. Thus, the components of the embedding vectors can be considered independent at least with this lag. This result is consistent with a similar computation performed on a daily time series (Lawrence et al. 1995). For a comparison, in Zhang (1996) the computation of the autocorrelation function for the period: $1850-1994$ gives a lag equal to 35 .

Methods of nonlinear dynamics can be strongly limited by typical features of experimental situations. In real cases it can happen that the presence of broadband noise can be 


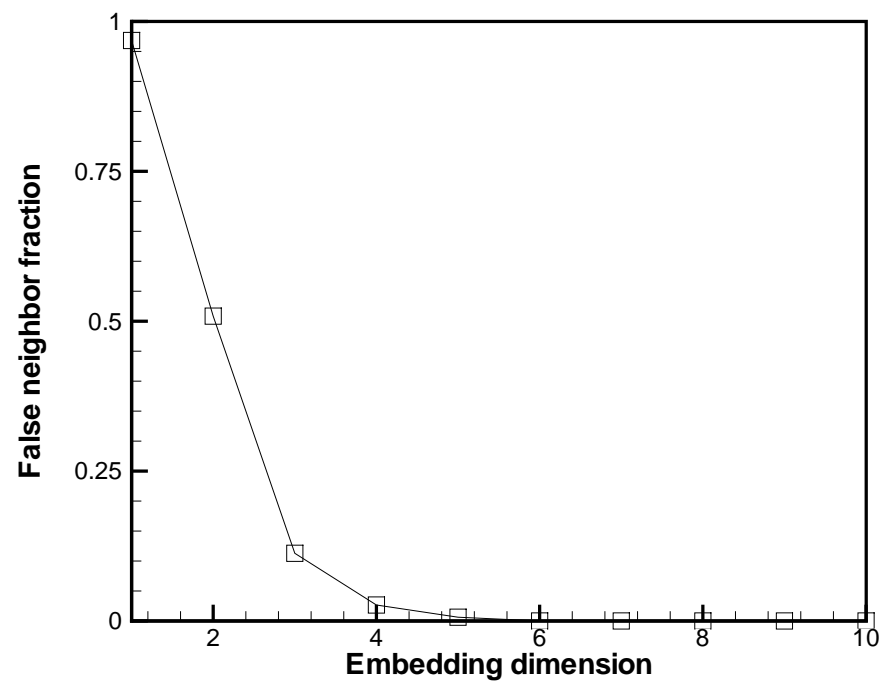

Fig. 4. False neighbor analysis: the minimum embedding dimension for monthly mean sunspot numbers is: $m=5$.

a severe pitfall for correlation dimension algorithms, compromising the reliability of distinction between stochastic and deterministic behaviour. In particular, our attempts to extrapolate a reliable estimate of correlation dimension for the original and filtered monthly mean sunspot numbers, using the most efficient methods available, failed due to the lack of a clear accurate saturation. Besides correlation dimensions the spectrum of Lyapunov exponents provides an important quantitative measure of the sensitivity to initial conditions. If the Lyapunov spectrum contains at least one positive exponent, then the corresponding system is chaotic and the value of that exponent is related to the magnitude of predictability time scale. However there are many difficulties to overcome for a reliable estimation of the whole Lyapunov spectrum from complex experimental data (Bryant 1990). Here we use a combined method derived from works by Sano \& Sawada (1985), Zeng et al. (1991), Brown et al. (1991). Let the Lyapunov exponents be arranged in decreasing order: $\lambda_{1} \geq \lambda_{2} \geq \ldots$ Using the concepts of "local" and "global" dimensions, estimated by correlation dimension computations, we determine a proper cut-off value for the number of exponents which can be related to the Lyapunov dimension. In fact, based on Kaplan-Yorke assumption, we compute the Lyapunov dimension as (Shuster 1984):

$D_{\mathrm{L}}=r+\frac{\sum_{i=1}^{r} \lambda_{i}}{\left|\lambda_{r+1}\right|}$

where: $r$ is the maximum number of exponents needed before the summation becomes negative. Here, for the computation of the full Lyapunov spectrum, we selected as local dimension: $d_{\mathrm{L}}=5$; while the optimal value for the global dimension was: $d_{\mathrm{G}}=2 d_{\mathrm{L}}+1=11$, for the monthly mean sunspot numbers.

In Fig. 5 we show the result of the full Lyapunov spectrum computation: the time relaxation of Lyapunov exponents is sufficient to extrapolate quite reliable estimates. For the monthly sunspot numbers, we found two positive exponents: $\lambda_{1}=0.106$ years $^{-1}$, and $\lambda_{2}=0.036$ years $^{-1}$ suggesting a real (hyper) chaotic motion with a limit for reliable deterministic predictions. The sum of all the positive Lyapunov exponents gives an estimation of the Kolmogorov entropy and its inverse, multiplied by $\log (2)$, gives the error doubling predictability time, $t_{\mathrm{p}}$. Thus, in our case, we found: $t_{\mathrm{p}}=4.85$ years (or $58 t_{\mathrm{s}}$ ). This time is the practical limit for reliable predictions. For comparison, in Zhang (1996) the evaluation of the positive Lyapunov exponent, through the method of Wolf et al. (1985), corresponds to a limit for deterministic prediction of about 3.6 years. These results are consistent with a more qualitative estimation of Lyapunov exponent ranging from 0.05 to 0.5 years $^{-1}$ given in Rozelot (1995). Finally, Lyapunov dimension estimation, based on Kaplan-Yorke conjecture Eq. (3), gives: $D_{\mathrm{L}} \approx 4.13$. The above result, clearly indicate a higher degree of geometrical complexity in the phase space for solar activity than that found in Zhang (1996) or in Lawrence et al. (1995) utilizing a correlation dimension analysis $(d \approx 2.8$ or $d \approx 2.3)$.

\section{Solar activity predictions}

The above complete characterisation of the nonlinear dynamics governing the monthly mean sunspot numbers allows us to construct a prediction model based on nonlinear deterministic behaviour of the related embedding vectors. Here we follow essentially the approach proposed in Farmer (1987) and suggested in Zhang (1996) to define a smooth map for the inverse problem. More precisely, the nonlinear deterministic behaviour in the embebdding space implies the existence of a smooth map: $f^{T}$ satisfying the relation:

$f^{T}\left(\boldsymbol{y}_{t}\right)=\boldsymbol{y}_{t+T}$

for a given embedding vector: $\boldsymbol{y}$. The inverse problem consists of the computation of this smooth map, given a scalar time series: $x(t), t=1, \ldots, n$. This map is the basis of our prediction model. Following the approach given in Zhang (1996) we first divided the known time series into two parts; the first one: $x(t), t=1, \ldots, n^{\prime}$ is used to set up the smooth map $f^{T}$, and the other part: $x(t), t=n^{\prime}+1, \ldots, n$ is used to check the accuracy of the prediction model. From the above nonlinear analysis we set: $n^{\prime}=n-\frac{t_{\mathrm{p}}}{t_{\mathrm{s}}}$. In order to calculate the unknown smooth function in Eq. (4) we assume a local linear hypothesis for the evolution of the embedding vectors, and this is quite safe for $T=1$. Given the last embedding vector, we selected the first $\boldsymbol{k}$ neighboring vectors near the reference vector in the $m=5$ embedding space, using a distance function. Then, we assumed that the evolution of the selected vector is correlated with the evolution of the neighboring vectors and the parameters of this correlation are computed through the solution of a proper least squares problem in the embedding space. More precisely, the order of the matrix for the least squares problem is given by $(\boldsymbol{k} \times m+1)$, and the predicted one-step ahead vector is given by solving the 




Fig. 5. Convergence behaviour of Lyapunov Spectrum for monthly mean sunspot numbers: two positive exponents suggest a genuine chaotic dynamic.

least squares problem for each component of the related $\boldsymbol{k}$ neighboring vectors:

$\boldsymbol{y}_{t+1}^{\prime(i)}=\alpha_{0}+\sum_{j=1}^{m} \alpha_{j} \boldsymbol{y}_{t}^{(j)} \quad$ for $\quad i=1, \ldots, m$.

This procedure is iterated for all the successive $n-n^{\prime}$ embedding vectors and the accuracy of the prediction model is estimated through the computation of the global average prediction error:

$<E^{2}>(f, \boldsymbol{k})=\frac{1}{\left(n-n^{\prime}\right)} \sum_{t=n^{\prime}+1}^{n}\left(\boldsymbol{y}_{\boldsymbol{t}}^{\prime}-\boldsymbol{y}_{\boldsymbol{t}}\right)^{2} / \sigma^{2}$.

The optimal model corresponds to the minimum value of $<E^{2}>(f, \boldsymbol{k})$ as a function of $\boldsymbol{k}$. The whole analysis is then performed for each new value added to the unknown part of the time series. As an example, the distribution of the optimal $\boldsymbol{k}$ values for the prediction of monthly mean sunspot numbers in the time interval limited by $t_{\mathrm{p}}$ is shown in Fig. 6.

The original time series, used for prediction analysis, is the noisy monthly mean sunspot numbers derived from SIDC archive, for the period: 1749.5-January 1999. At this last time, the current 23rd cycle results were well developed (more than two years after the new cycle minimum) and thus we are able to test the efficiency and accuracy of all the advanced prediction methods. We recall that the final prediction is related to the filtered values for the monthly smoothed sunspot numbers, computed using the relation:

$\tilde{S_{n}}=\frac{1}{12}\left[\sum_{k=n-5}^{n+5} S_{k}+\frac{1}{2}\left(S_{n+6}+S_{n-6}\right)\right]$

where $S_{k}$ is the mean value of $S$ for the month $k$. This choice is motivated by the fact that, even if the monthly

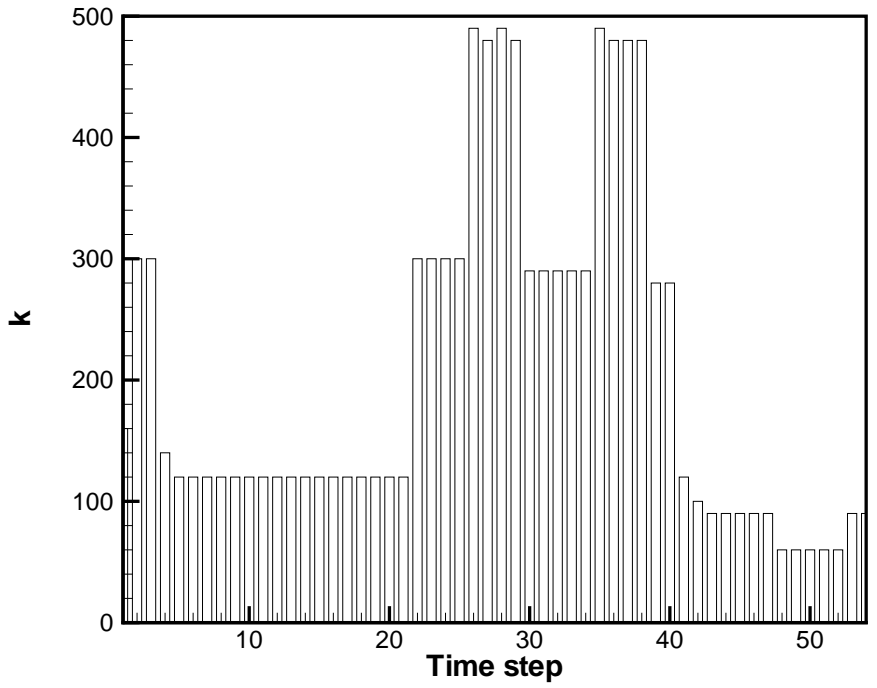

Fig. 6. Optimal distribution for neighbor vector number, $\boldsymbol{k}$, in the nonlinear method as a function of prediction time steps.

mean sunspot numbers contain a higher level of broadband noise which can severely degrade the accuracy of predictions, smoothing in principle is not an invariant process in dynamical systems and may affect some intrinsic features of the original data (Palus 1995). Figure 7 shows the results of the nonlinear prediction model (solid line) for a period limited by the error doubling predictability time, $t_{\mathrm{p}}=2003.3$. As we can see the maximum smoothed value for the 23rd cycle is predicted near April (or May) 2000 with the value: $125.6 \pm 7.1$. Error bars show the uncertainty of predicted values. Based on this prediction, the value is comparable with the maximum reached in 1937(119.2).

To compare, a posteriori, the accuracies of predictions obtained using the most efficient methods proposed in the literature, in the same figure are shown the observed data 


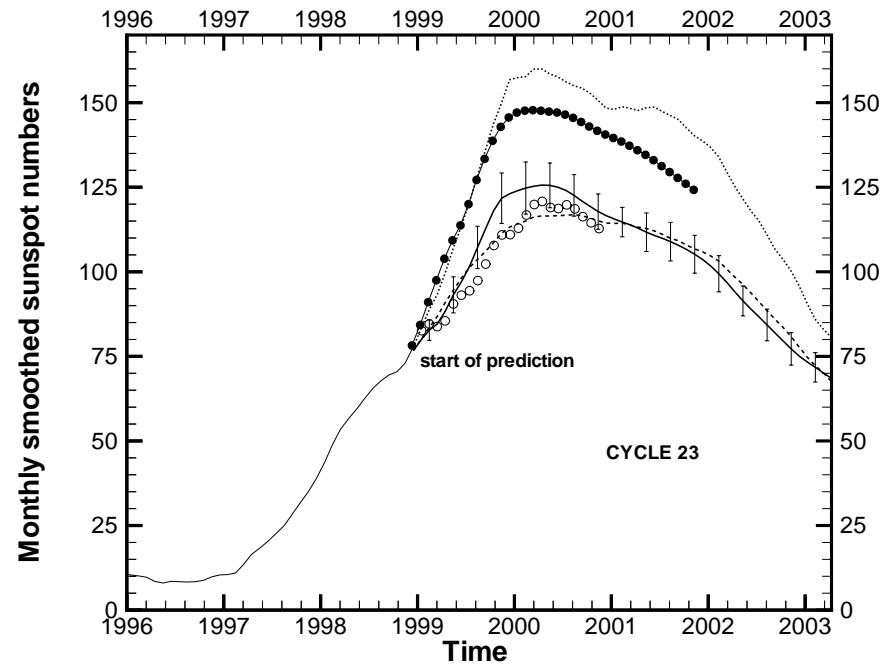

Fig. 7. Nonlinear forecasting (solid line with error bars) vs. other prediction methods. Line-points: original Zhang nonlinear method. Open circles: observed data.

from SIDC (circles); the original nonlinear method by Zhang (1996) (line-points); the precursor method based on solar and geomagnetic activity from the IPS archive (Thompson 1999) (dotted line); and the prediction from the modified method of McNish-Lincoln, using regression coefficients and mean cycle values computed for cycles 8 through 20, from SIDC archives (dashed line) (National Geophysical Data Center, NOAA). A qualitative analysis indicates a good coherence of these methods to predict the phase of the next maximum, but with significative differences in the amplitude. At present we can reasonably assume that the monthly smoothed sunspot numbers, for the 23rd solar cycle, peaked at April 2000 with the amplitude value: $\approx 120.8$. As we can see, the nonlinear method correctly predicted this result, inside the uncertainty interval. Another prediction method reached the same absolute accuracy for the amplitude value: the modified method of McNish-Lincoln. Two other methods are included in the comparative analysis: the prediction method based on an interpolation of Waldmeier's standard curves (SIDC classical method); and the combined method of Denkmayr (1997) and Hanslmeier et al. (1999), a nonparametric regression technique coupling a dynamo-based geomagnetic estimator with Waldmeier's idea of standard curves. Note that typical precursor methods, geomegnetic and solar, predicted higher amplitudes than average with maximum values around 160-168 with an uncertainty of about 15\% (Joselyn et al. 1997; Lantos \& Richard 1998). Moreover, these high values are consistent with many predictions from univariate or bivariate, purely statistical methods which correlate geomagnetic indices with annual sunspot numbers. Equivalent high peak values can be derived from typical neural network approaches, which appear quite consistent with solar dynamo predictions. In order to complete our comparison analysis, we applied the original Zhang nonlinear method using the indication and parameter values suggested in his original paper

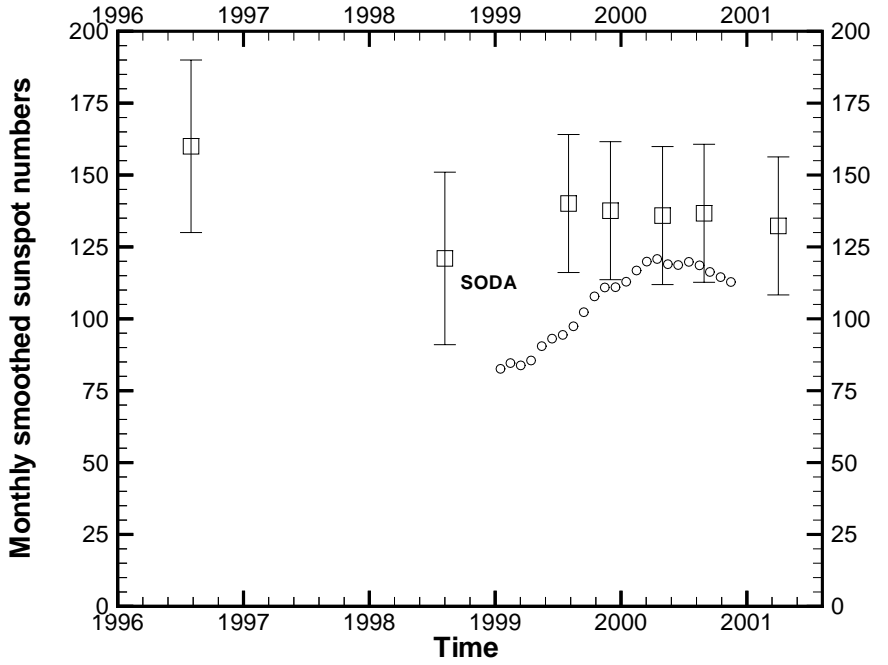

Fig. 8. Changes in predicted maximum amplitude for a geomagnetic precursor method as the 23rd cycle progresses (from MSFC, NASA archive) Circle symbols: observed data.

(Zhang 1996), where we obtained quite good predictions for the descending part of the previous (22nd) cycle. More precisely, here we set a delay time $\tau=35$ from the autocorrelation analysis; a dimension $d=3$ for the embedding space; an optimal $k=380$ for the nearest neighboring phase points; and finally we corrected the predictions using the exponential divergence formula for embedding space trajectories with the largest Lyapunov exponent estimated as: $\lambda_{1}=0.276$ years $^{-1}$. From Fig. 7 we can note that the results given by original Zhang method are quite similar to those of typical precursor methods.

Figure 8 shows the adjustments of the predicted maximum amplitude, as the $23 \mathrm{rd}$ cycle progresses for a geomagnetic precursor method developed by the Nasa Marshall Space Flight Center team (Hathaway et al. 1999). This allows one to evaluate the degree of reliability of the basic physical hypothesis of current precursor- like methods which correlate (using a linear model) the changes in the Earth's magnetic field or the size of the sun's polar magnetic fields near the minimum, with the future solar activity. Based on solar dynamo predictions and the Solar Dynamo Amplitude (or SODA) index, which is a purely solar indicator, Schatten et al. (1996), Sofia et al. (1998), provided mildly smaller values: $143 \pm 30$ or $121 \pm 30$ in early 2000, for maximum smoothed sunspot number. Circles show the observed smoothed sunspot numbers.

As a forecast evaluation, a quantitative analysis, based on a global mean prediction error, Eq. (6), for assumed unknown values after January 1999 and updated observations to May 2001, is summarized in Table 1. In this way we are able to estimate the accuracy of the solar cycle shape predictions near maximum. Besides this normalized mean square error, which is a measure of forecast accuracy, or the mean degree of correspondence between individual pairs of forecasts and observations, in the same table we show the forecast reliability or bias, as measured by the normalized mean error, and the linear correlation 
Table 1. Prediction models: global forecast evaluation.

\begin{tabular}{lccc}
\hline Model & $\left\langle E^{2}>\right.$ & bias & corr \\
\hline McNish-Lincoln modified & 0.08 & 0.01 & 0.94 \\
Nonlinear & 0.34 & 0.04 & 0.93 \\
Waldmeier standard curves & 0.56 & 0.01 & 0.64 \\
Combined method (Denkmayr) & 1.85 & 0.10 & 0.91 \\
Nonlinear (Zhang 1996) & 5.12 & 0.18 & 0.92 \\
Precursor (Thompson, IPS) & 8.12 & 0.22 & 0.93 \\
\hline
\end{tabular}

coefficient, a measure of the linear association between the forecasts and observations (Doggett 1993).

If we assume that the current 23rd solar cycle peaked during the year 2000 with a yearly mean of 119.6 , the best forecast performance is obtained by the modified method of McNish-Lincoln, followed by the nonlinear method. Moreover, our version of the original Zhang nonlinear method shows better performances, as clearly indicated in Table 1. The accuracy of geomagnetic precursor-like methods and NOAA panel forecasts was quite low, suggesting the necessity of more refinements in the basic solar dynamo models used, in particular the introduction of more significant nonlinear and stochastic contributions. The predicted peak using the SODA index appears more accurate but the uncertainty interval is too high, mainly due to difficulties in reaching accurate magnetic field measurements. The good performance of the modified McNish-Lincoln method can be explained here considering the slow ascending phase of the current cycle, correctely covered by the average characteristics of the past cycles. A different situation occurred in the previous cycle, as clearly shown by Fessant et al. (1996), where the predictions for its anomalously fast ascending phase with a neural network approach resulted superior predictions. Another nonlinear method by Verdes et al. (2000), based on the correlation between the second derivative of proper smoothed data at cycle minimum and the next maximum amplitude, predicted a peak of $115 \pm 32$. Moreover, a neural network approach for yearly mean data predicted a higher mean value: $135 \pm 17$, consistent with most precursor methods. Of course we need to wait until the end of the descending phase of the current cycle to draw a complete picture. Our result completely reverse the conclusion stated in a recent work by Li et al. (2001) where the authors show the overall superiority of precursor-like methods to non-precursor ones, analyzing a wide collection of yearly and monthly mean predictions of maxima for solar cycles 21 to 23. A recent work by Lantos (2000) partially supports our scenario, predicting a low peak of $103 \pm 20$ using a method based on the slope at the inflexion point observed during the ascending phase of the cycle. The residual errors, detected in the nonlinear method, are mainly due to the presence of high-amplitude stochastic fluctuations in solar activity not included in the model, as recently described by Charbonneau \& Dikpati (2000). A possible way to quantify the importance of these stochastic components, for solar cycle predictions, is the analysis of the wavelet entropy, a proper wavelet functional applied to solar cycles which quantifies the degree of frequency energy dispersion (Sello 2000). In fact, the current wavelet entropy behaviour suggests an increasing high solar cycle complexity level, indicating the presence of some high stochastic fluctuation contributions for the solar photospheric dynamics.

\section{Conclusions}

The problem of the monthly smoothed sunspot number prediction is examined with particular attention to a nonlinear dynamic approach. The intrinsic complexity of the related time series strongly affects the accuracy of the phase and amplitude predictions. Starting from a recent work, we checked the reliability of a forecasting model based on concepts of nonlinear dynamics theory applied to experimental time series, like embedding phase space, Lyapunov spectrum and chaotic behaviour. The analysis clearly pointed out the nonlinear-chaotic nature, with limited predictability, for the solar activity as suggested in many previous works. The model is based on a local hypothesis of the behaviour on the embedding space, utilizing an optimal number of neighbor vectors to correlate the future evolution of the current point. The characteristic parameters of the model are triggered through an extensive parametric computation. The main task was to set up and to compare, using the observed data for the current 23rd solar cycle, this nonlinear prediction technique (numerically based on an inverse problem), with the current most reliable prediction methods, especially the so-called precursor methods, physically based on solar dynamo theory, and proposed to reach accurate long-term predictions of solar activity. A detailed quantitative forecast evaluation for different methods, including the original nonlinear method by Zhang (1996), shows that the best global performance is obtained by the so-called modified method of McNish-Lincoln, followed by our nonlinear prediction technique. Geomagnetic precursor-like methods showed quite low accuracies suggesting the necessity for more refined analyses in order to better investigate the basic assumptions and methodologies of these techniques, especially the solar dynamo-based models used and their significant nonlinear components. The overall good performance of the nonlinear method is further supported considering a residual uncertainty due to the presence of high-amplitude stochastic fluctuations in current solar activity, which can be evaluated by the wavelet entropy behaviour.

\section{References}

Abarbanel, H. D. I., Brown, R., \& Kadtke, J. B. 1990, Phys. Rev. A, 41, 4

Brown, R., Bryant, P., \& Abarbanel, H. D. I. 1991, Phys. Rev. A, 43,6

Bryant, P., Brown, R., \& Abarbanel, H. D. I. 1990, Phys. Rev. Lett., 65,13 
Calvo, R. A., Ceccatto, H. A., \& Piacentini, R. D. 1995, AJ, Mundt, M. D., Maguire, W. B., \& Chase, R. R. P. 1991, J. 444,916

Carbonell, M., Oliver, R., \& Ballester, J. L. 1994, A\&A, 290, 983

Charbonneau, P., Dikpati, M. 2000, ApJ, 543, 2, 1027

Cugnon, P. 1999, Solar Influences data Analysis Center, Brussels, http://www.oma.be/KSB -ORB/SIDC/index.html Geophys. Res., 96, 1705

Palus, M. 1995, Physica D, 80, 186

Prichard, D., \& Theiler, J. 1994, Phys. Rev. Lett., 73, 951

Price, C. P., Prichard, D., \& Hogeson, E. A. 1992, J. Geophys. Res., 97, 19

Rozelot, J. P. 1995, A\&A, 297, L45

Sano, M., \& Sawada, Y. 1985, Phys. Rev. Lett., 55, 10

Denkmayr, K, \& Cugnon, P. 1997, in Solar-Terrestrial Prediction Workshop V

Schatten, K. H., Scherrer, P. M., Svalgaard, L., \& Wilcox, J. M. 1978, Geophys. Res. Lett., 5, 411

Doggett, K. A. 1993, in Solar-Terrestrial Predictions IV, ed. Hruska et al., Proc. of a workshop at ottawa (Canada), 1

Farmer, J. D., \& Sidorowich, J. J. 1987, Phys. Rev. Lett., 59, 845

Fessant, F., Pierret, C., \& Lantos, P. 1996, Solar Phys., 168, 423

Fraser, A. M., \& Swinney, H. L. 1986, Phys. Rev. A, 33, 2

Grassberger, P., \& Procaccia, I. 1983, Physica D, 9

Hanslmeier, A., Denkmayr, K., \& Weiss, P. 1999, Solar Phys., 184,1

Hathaway, D., Wilson, R. M., \& Reichmann, E. J. 1999, J. Geophys. Res., 104

Joselin, J. A., Anderson, J., Coffey, H., et al. 1997, EOS Trans. Amer. Geophys. Union, 78, 211

Kennel, M. B., \& Abarbanel, H. D. I. 1994, Phys. Rev. E, 47, 4

Kugiumtzis, D. 1999, Los Alamos National Laboratory Preprint Archive, submitted to Phys. Rev. E [Physics/9905021]

Kulkarni, D. R., Pandya, A. S., \& Parikh, J. C. 1998, Geophys. Res. Lett., 25, 4, 457

Lantos, P., \& Richard, O. 1998, Solar Phys., 182, 1

Lantos, P. 2000, Solar Phys., 196, 1

Lawrence, J. K., Cadavid, A. C., \& Ruzmaikin, A. A. 1995, ApJ, 455

Li, L. H., \& Sofia, S. 2001, ApJ, 549, 2, 1204

Li, K. J., Yun, H. S., \& Gu, X. M. 2001, A\&A, 368, 1, 285

McNish, A. G., \& Lincoln, J. V. 1949, Trans. Am. Geophys. Union, 30, 673

Schatten, K. H., \& Pesnell, W. D. 1993, Geophys. Res. Lett., 20,2275

Schatten, K., \& Sofia, S. 1996, Am. Astr. Soc. Meet., 189

Schreiber, T. 1998, Phys. Rep., 308

Schuster, H. G. 1984, Deterministic Chaos, ed. Physik-Verlag

Sello, S. 1999, Los Alamos National Laboratories Preprint Archive [Physics/9901050]

Sello, S. 2000, A\&A, 363, 1, 311

Sofia, S., Fox, P., \& Schatten, K. 1998, Geophys. Res. Lett., 25,22

Spiegel, E. A., Zhan, J. P. 1996, Technical Report (Columbia University New York)

Thompson, R. J. 1993, Solar Phys., 148, 383

Thompson, R. 1999, IPS Radio and Space Services, Sydney http://www.ips.gov.au/asfc/curren t/solar.html

Theiler, J. 1990, J. Opt. Soc. Am. A, 7, 6

Verdes, P. F., Parodi, M. A., Granitto, P. M., et al. 2000, Solar Phys., 191(2), 421

Wolf, A., Swift, J. B., Swinney, H. L., \& Vastano, J. A. 1985, Physica D, 16, 285

Zeng, X., Eykholt, R., \& Pielke, R. A. 1991, Phys. Rev. Lett., 66,25

Zhang, Q. 1996, A\&A, 310, 646

Zhang, Q. 1994, Acta Astron. Sin., 35

Zhang, Q. 1995, Acta Astron. Sin., 15 